\title{
A Remark on the Rank of Positive Semidefinite Matrices Subject to Affine Constraints*
}

\author{
A. Barvinok \\ Department of Mathematics, University of Michigan, \\ Ann Arbor, MI 48109-1109, USA \\ barvinok@math.1sa.umich.edu
}

\begin{abstract}
Let $\mathcal{K}_{n}$ be the cone of positive semidefinite $n \times n$ matrices and let $\mathcal{A}$ be an affine subspace of the space of symmetric matrices such that the intersection $\mathcal{K}_{n} \cap \mathcal{A}$ is nonempty and bounded. Suppose that $n \geq 3$ and that $\operatorname{codim} \mathcal{A}=\left(\begin{array}{c}r+2 \\ 2\end{array}\right)$ for some $1 \leq r \leq n-2$. Then there is a matrix $X \in \mathcal{K}_{n} \cap \mathcal{A}$ such that rank $X \leq r$. We give a short geometric proof of this result, use it to improve a bound on realizability of weighted graphs as graphs of distances between points in Euclidean space, and describe its relation to theorems of Bohnenblust, Friedland and Loewy, and Au-Yeung and Poon.
\end{abstract}

\section{Introduction}

Let $S_{y} m_{n}$ be the space of $n \times n$ symmetric matrices. Thus $S y m_{n}$ is a real vector space of dimension $\left(\begin{array}{c}n+1 \\ 2\end{array}\right)$. Let $\mathcal{K}_{n} \subset S y m_{n}$ be the convex cone of positive semidefinite matrices. The following result is well known, see, for example, Barvinok (1995), Section 31.5 of Deza and Laurent (1997), and Pataki (1996).

(1.1) Theorem. Let $\mathcal{A} \subset S y m_{n}$ be an affine subspace such that the intersection $\mathcal{K}_{n} \cap \mathcal{A}$ is nonempty and $\operatorname{codim} \mathcal{A} \leq\left(\begin{array}{c}r+2 \\ 2\end{array}\right)-1$ for some nonnegative integer $r$. Then there is a matrix $X \in \mathcal{K}_{n} \cap \mathcal{A}$ such that $\operatorname{rank} X \leq r$.

The bound in Theorem 1.1 is sharp, meaning that for any $n$ and for any $r<n$ one can construct an affine subspace $\mathcal{A}$ such that $\mathcal{A} \cap \mathcal{K}_{n} \neq \emptyset, \operatorname{codim} \mathcal{A}=\left(\begin{array}{c}r+2 \\ 2\end{array}\right)$ and for every $X \in \mathcal{K}_{n} \cap \mathcal{A}$ one has rank $X>r$ (see Section 2).

\footnotetext{
* This research was partially supported by NSF Grant DMS 9734138.
} 
The problem of finding a low-rank positive semidefinite matrix subject to affine constraints has attracted some attention recently. For example, it turned out to be relevant to problems of chemistry, statistics, archaeology, genetics, and geography (see Alfakih and Wolkowicz (1998) for references and discussion) and to semidefinite programming in general, currently a very active area of research (see, for example, Vandenberghe and Boyd (1996) for a survey). Therefore, it is worth mentioning that in one particular case the bound can be sharpened.

(1.2) Theorem. Suppose that $r>0$ and $n \geq r+2$. Let $\mathcal{A} \subset$ Sym $_{n}$ be an affine subspace such that codim $\mathcal{A}=\left(\begin{array}{c}r+2 \\ 2\end{array}\right)$. Suppose that the intersection $\mathcal{K}_{n} \cap \mathcal{A}$ is nonempty and bounded. Then there is a matrix $X \in \mathcal{K}_{n} \cap \mathcal{A}$ such that $\operatorname{rank} X \leq r$.

Theorem 1.2 is equivalent to a host of known results in Matrix Theory (see Section 4), yet it appears that it has not been stated explicitly. The purpose of this note is to provide a direct proof of the theorem based solely on the convex geometry of $\mathcal{K}_{n}$ (Section 3), to compare Theorems 1.1 and 1.2 from the structural (this section) and algorithmic points of view (Section 2), and to use Theorem 1.2 for a problem of Distance Geometry (Section 2).

Polyhedral Analogy. Theorem 1.1 is proved by pointing out that every extreme point of the intersection $\mathcal{K}_{n} \cap \mathcal{A}$ will have the desired rank (see Section 31.5 of Deza and Laurent, 1997). The proof can also be obtained via complementarity conditions for semidefinite programming (see Barvinok, 1995; Alizadeh et al., 1997), which constitute a particular case of the complementary conditions for general linear programs (see Anderson and Nash, 1987). Thus Theorem 1.1 is somewhat analogous to the statement that the intersection of an affine subspace of codimension $r$ with a nonnegative orthant in $\mathbb{R}^{n}$, if nonempty, will contain a point where some $n-r$ coordinates are zero (see, for example, Pataki (1998) for a discussion of this "polyhedral analogy" and based on it a semidefinite version of the simplex method). Furthermore, for a generic subspace the bound $n-r$ cannot be improved. In the context of Theorem 1.2, it is not true that every extreme point of the intersection will have the desired rank, but rather that the rank of some extreme point will satisfy the bound. Theorem 1.2 demonstrates a point where the polyhedral analogy breaks down. Essentially, it asserts that there is no positive semidefinite analogue of a simple bounded polyhedron (simple polytope) as there must always be a degenerate vertex.

\section{An Application to Distance Geometry}

We fix an undirected weighted graph $G=(V, E ; \rho)$ with the set $V=\{1, \ldots, n\}$ of vertices, a set $E \subset\left(\begin{array}{c}V \\ 2\end{array}\right)$ of edges and nonnegative weights $\rho_{i j}$ on the edges. Let $r$ be a positive integer. We say that $G$ is $r$-realizable if $G$ can be realized as the graph of distances between points in Euclidean space $\mathbb{R}^{r}$, that is, if we can find $n$ (not necessarily distinct) points $v_{1}, \ldots, v_{n}$ in $\mathbb{R}^{r}$ such that $\left\|v_{i}-v_{j}\right\|=\rho_{i j}$ whenever $\{i, j\} \in E$. The problem of finding such a realization is known as the Euclidean distance matrix completion problem, see Laurent (1998) for a survey. We use two reformula- 
tions of the problem, the first one is more "straightforward" while the second is more "economical."

(2.1) First Reformulation. Let $G=(V, E ; \rho)$ be a weighted graph on $n$ nodes and let $v_{1}, \ldots, v_{n} \in \mathbb{R}^{r}$ be its realization. We consider the Gram matrix $X=\left(\xi_{i j}\right)$ of $v_{1}, \ldots, v_{n}$, so $\xi_{i j}=\left\langle v_{i}, v_{j}\right\rangle$, where $\langle\cdot, \cdot\rangle$ is the standard scalar product in $\mathbb{R}^{n}$. Then $X$ is a positive semidefinite matrix such that $\operatorname{rank} X \leq r$. Furthermore, the distance conditions

$$
\left\|v_{i}-v_{j}\right\|=\rho_{i j} \quad \text { for } \quad\{i, j\} \in E
$$

can be written as $|E|$ affine constraints

$$
\xi_{i i}-2 \xi_{i j}+\xi_{j j}=\rho_{i j}^{2} \quad \text { for } \quad\{i, j\} \in E .
$$

Conversely, if $X=\left(\xi_{i j}\right)$ is an $n \times n$ positive semidefinite matrix satisfying (2.1.1) and such that rank $X \leq r$, then $X$ can be written in the form $\xi_{i j}=\left\langle v_{i}, v_{j}\right\rangle$, where $v_{1}, \ldots, v_{n} \in \mathbb{R}^{r}$ is an $r$-realization of $G$. Let $\mathcal{A}_{n} \subset S y m_{n}$ be the affine subspace of matrices satisfying (2.1.1). We conclude that $G$ is $r$-realizable if and only if there exists a matrix $X \in \mathcal{K}_{n} \cap \mathcal{A}_{n}$ such that rank $X \leq r$.

(2.2) Second Reformulation. We observe that we can always translate the vertices $v_{1}, \ldots, v_{n}$ of a realization of $G$ so that $v_{n}=0$. Let $X=\left(\xi_{i j}\right)$ be the $(n-1) \times(n-1)$ Gram matrix of the vectors $v_{1}, \ldots, v_{n-1}$, so $\xi_{i j}=\left\langle v_{i}, v_{j}\right\rangle$. Then (2.1.1) can be replaced by $|E|$ affine constraints

$$
\begin{aligned}
\xi_{i i} & =\rho_{i n}^{2} \quad \text { if } \quad\{i, n\} \in E \quad \text { and } \\
\xi_{i i}-2 \xi_{i j}+\xi_{j j} & =\rho_{i j}^{2} \quad \text { if } \quad 1 \leq i, j \leq n-1 \quad \text { and } \quad\{i, j\} \in E
\end{aligned}
$$

so that $G$ is $r$-realizable if and only if there is an $(n-1) \times(n-1)$ positive semidefinite matrix $X$ satisfying (2.2.1) and such that rank $X \leq r$. Let $\mathcal{A}_{n-1} \subset S y m_{n-1}$ be the affine subspace of matrices satisfying (2.2.1). We conclude that $G$ is $r$-realizable if and only if there exists a matrix $X \in \mathcal{K}_{n-1} \cap \mathcal{A}_{n-1}$ such that $\operatorname{rank} X \leq r$.

The following result was proved in Barvinok (1995).

(2.3) Theorem. Suppose that the number $|E|$ of edges of a weighted graph $G=$ $(V, E ; \rho)$ satisfies the inequality $|E| \leq\left(\begin{array}{c}r+2 \\ 2\end{array}\right)-1$. Then $G$ is realizable in $\mathbb{R}^{r}$ if and only if $G$ is realizable in some Euclidean space.

Proof. Follows by Reformulation 2.1 and Theorem 1.1 .

The following example shows that the bound in Theorem 2.3 and hence in Theorem 1.1 is indeed sharp.

(2.4) Example. Suppose that $G$ consists of a complete graph on the first $r+2$ vertices (1-skeleton of an $(r+1)$-dimensional simplex $)$ and $n-r-2$ isolated vertices. We assign 
weight 1 to every $\left(\begin{array}{c}r+2 \\ 2\end{array}\right)$ edge of $G$. Clearly, any realization of $G$ as a graph of distances in some space $\mathbb{R}^{d}$ amounts to placing the first $r+2$ vertices as the vertices of a regular $(r+1)$-dimensional simplex and then placing arbitrarily the remaining $(n-r-2)$ vertices. Thus $G$ is realized in $\mathbb{R}^{r+1}$ but not realized in $\mathbb{R}^{r}$.

Theorem 1.2 allows us to prove that in the critical case of $|E|=\left(\begin{array}{c}r+2 \\ 2\end{array}\right)$, the graph from Example 2.4 is the only graph that defies the conclusion of Theorem 2.3.

(2.5) Theorem. Suppose that $|E|=\left(\begin{array}{c}r+2 \\ 2\end{array}\right)$ for some $r>0$ and that $G$ is not a union of a complete graph with $r+2$ vertices and zero or more isolated vertices. Then $G$ is realizable in $\mathbb{R}^{r}$ if and only if $G$ is realizable in some Euclidean space.

Proof. Since $G$ is $r$-realizable if and only if its connected components are realizable, without loss of generality we may assume that $G$ is connected. Since $G$ is not a complete graph, for the number $n$ of vertices we have $n \geq r+3$. We now use Reformulation 2.2 to conclude that the $r$-realizability of $G$ is equivalent to the existence of a matrix $X \in$ $\mathcal{K}_{n-1} \cap \mathcal{A}_{n-1}$ such that rank $X \leq r$, where $\mathcal{A}_{n-1} \subset S y m_{n-1}$ is the affine subspace defined by (2.2.1). We prove that the intersection $\mathcal{A}_{n-1} \cap \mathcal{K}_{n-1}$ is necessarily bounded. Since $G$ is connected, each vertex $i$ of $G$ can be connected to the $n$th vertex by a path in the graph. Therefore, for any realization of $G$ with $v_{n}=0$, the length $\left\|v_{i}\right\|$ is bounded by the sum of the lengths of the edges of the path, so we can write $\left\|v_{i}\right\| \leq R$, where $R$ is the sum of all weights $\rho_{i j}$. Hence $\left|\xi_{i i}\right| \leq R^{2}$. Now we observe that the set of positive semidefinite matrices with a uniform bound on the diagonal entries is bounded and apply Theorem 1.2.

Example 2.4 provides evidence that both conditions $\mathcal{K}_{n} \cap \mathcal{A} \neq \emptyset$ and $n \geq r+2$ in Theorem 1.2 are necessary. Indeed, consider Reformulation 2.2 of the $r$-realizability problem for graph $G$ of Example 2.4. If $n=r+2$ (there are no isolated vertices), then the condition on the size of the matrix is violated (remember that we pass to $(n-1) \times(n-1)$ matrices). If $n>r+2$ and hence there are isolated vertices, then the "realization space" $\mathcal{K}_{n-1} \cap \mathcal{A}_{n-1}$ is not bounded, because an isolated vertex can move freely.

Algorithmic Issues and Mechanical Analogies. It is relatively easy to find, at least approximately, a matrix $X$ satisfying the conditions of Theorem 1.1. In Barvinok (1995), the following approach was suggested. Choose a positive definite matrix $F$ and find a positive semidefinite matrix $X$ from the intersection $\mathcal{K}_{n} \cap \mathcal{A}$ which minimizes $\operatorname{tr}(F X)$ (this is a problem of semidefinite programming (see Vandenberghe and Boyd, 1996). It is shown in Barvinok (1995) that if $F$ is sufficiently generic, then the matrix $X$ satisfies the required rank condition. This method has an especially transparent interpretation for realizability problems discussed in this section. Given a higher-dimensional realization of a weighted graph, we attach "springs" (with generic elasticities) to all pairs of vertices that are not connected by an edge. Then the graph will fold itself down onto the minimal energy configuration of an appropriate dimension. In Alfakih 
and Wolkowicz (1998) it is shown how to avoid a nonconstructive genericity condition on $F$. With any positive definite $F$, we find a matrix $X$ as above. If $X$ is an extreme point of $\mathcal{K}_{n} \cap \mathcal{A}$, it satisfies the desired rank condition. Otherwise, let $\mathcal{F}$ be a face of $\mathcal{K}_{n} \cap \mathcal{A}$ containing $X$. Then Alfakih and Wolkowicz (1998) show how to determine a direction in which to move from $X$ in $\mathcal{F}$ until reaching a point $Y$ of a smaller rank on the boundary of $\mathcal{F}$. Iterating this process, we get a matrix of the required rank.

In Theorem 1.2 the situation seems to be quite different. It is not clear how to construct the desired matrix $X$ efficiently. Neither the proof of this paper nor other approaches deducing Theorem 1.2 from known results in matrix theory (see Section 4) are constructive. For instance, it is not clear what would be the right "spring attachment" to obtain the $r$-dimensional realization in Theorem 2.5.

We state the problem of finding matrix $X$ in Theorem 1.2 efficiently as an open problem.

Finally, we mention that Theorems 1.1 and 1.2 can be applied in a similar way to other problems of geometric realizability involving constraints on lengths and angles, as long as those constraints can be recast as affine constraints on a Gram matrix.

(2.6) Example. Suppose we want to find a hexagon in $\mathbb{R}^{r}$ with prescribed lengths of its sides, prescribed angles between the three pairs of opposite sides, and prescribed sum of the squared norms of the vertices. These constraints can be written as 10 affine constraints on the $6 \times 6$ Gram matrix of vectors $v_{1}, \ldots, v_{6} \in \mathbb{R}^{r}$. Theorem 1.2 implies that such a hexagon exists in $\mathbb{R}^{r}$ if and only if it exists in $\mathbb{R}^{3}$.

\section{Proof}

Our proof uses the convex geometry of the cone $\mathcal{K}_{n}$ of positive semidefinite matrices.

Let $K \subset \mathbb{R}^{d}$ be a convex set. An affine hyperplane $H$ isolates $K$ if $K$ lies in one of the closed halfspaces bounded by $H$. A set $F \subset K$ is called a face of $K$ if there is a hyperplane $H$ which isolates $K$ and such that $F=K \cap H$. In this case, $H$ is called a supporting hyperplane of $F$. We also agree that $K$ is a face of itself.

By the interior int $K$ of a convex set $K$ we always mean its relative interior, that is the interior with respect to the affine hull aff $K$. The set $\partial K=K \backslash$ int $K$ is called the boundary of $K$. See Webster (1994) as a general reference.

Thus $\mathcal{K}_{n}$ is a pointed convex closed cone. We need a description of the facial structure of $\mathcal{K}_{n}$ (see Section 31.5 of Deza and Laurent, 1997).

(3.1) The Facial Structure of $\mathcal{K}_{n}$. The faces of $\mathcal{K}_{n}$ are parameterized by the subspaces of $\mathbb{R}^{n}$. For a subspace $\mathcal{L} \subset \mathbb{R}^{n}$, let

$$
\mathcal{F}_{\mathcal{L}}=\left\{X \in \mathcal{K}_{n}: \mathcal{L} \subset \operatorname{Nul} X\right\}
$$

where $\mathrm{Nul} X$ is the null space of $X$ consisting of vectors $y \in \mathbb{R}^{n}$ such that $X y=\mathbf{0}$. One 
has $\operatorname{dim} \mathcal{F}_{\mathcal{L}}=\left(\begin{array}{c}r+1 \\ 2\end{array}\right)$, where $r=\operatorname{codim} \mathcal{L}$. A matrix $X \in \mathcal{K}_{n}$ of rank $r$ lies in the interior of the $\left(\begin{array}{c}r+1 \\ 2\end{array}\right)$-dimensional face $\mathcal{F}_{\mathrm{Nul} X}$.

The face $\mathcal{F}_{\mathcal{L}}$ "looks like" the cone of positive semidefinite matrices of size $r$, where $r=\operatorname{codim} \mathcal{L}$. Decomposing $\mathbb{R}^{n}=\mathcal{L} \oplus \mathcal{L}^{\perp}$, we see that in an appropriate basis, every matrix $X \in \mathcal{F}_{\mathcal{L}}$ has the structure

$$
X=\left(\begin{array}{cc}
\mathbf{0}_{(n-r) \times(n-r)} & \mathbf{0}_{(n-r) \times r} \\
\mathbf{0}_{r \times(n-r)} & Y_{r \times r}
\end{array}\right),
$$

where $\mathbf{0}$ is the zero matrix and $Y_{r \times r}$ is an $r \times r$ positive semidefinite matrix. In particular, identifying $\mathbb{R}^{r}$ with the orthogonal complement $\mathcal{L}^{\perp}$ of $\mathcal{L}$, we identify $\mathcal{F}_{\mathcal{L}}$ with $\mathcal{K}_{r}$. In other words, there is a rank-preserving isometry $\mathcal{K}_{r} \longrightarrow \mathcal{F}_{\mathcal{L}}$, where $\operatorname{codim} \mathcal{L}=r$.

The boundary $\partial \mathcal{F}_{\mathcal{L}}$ is the union of all faces $\mathcal{F}_{\mathcal{M}}$, where $\mathcal{M} \subset \mathbb{R}^{n}$ is a subspace strictly containing $\mathcal{L}$.

The following lemma provides the key ingredient of the proof of Theorem 1.2.

(3.2) Lemma. Let $r \geq 1$ and let $\mathcal{A} \subset S y m_{r+2}$ be an affine subspace such that $\operatorname{dim} \mathcal{A}=$ $r+2$ and so $\operatorname{codim} \mathcal{A}=\left(\begin{array}{c}r+2 \\ 2\end{array}\right)$. Suppose that the intersection $\mathcal{A} \cap \mathcal{K}_{r+2}$ is nonempty and bounded. Then there is an $X \in \mathcal{K}_{r+2} \cap \mathcal{A}$ such that $\operatorname{rank} X \leq r$.

Proof. We observe that if $\mathcal{A} \cap$ int $\mathcal{K}_{r+2}=\emptyset$, then the separation theorem (see Webster, 1994) implies that $\mathcal{A}$ lies in the supporting hyperplane of a proper face $\mathcal{F}$ of $\mathcal{K}_{r+2}$. Since there is a rank-preserving isometry between $\mathcal{F}$ and $\mathcal{K}_{s}$ for some $s<r+2$ (see Section 3.1), we deduce the result from Theorem 1.1.

Hence without loss of generality we can assume that $\mathcal{A}$ contains an interior point of $\mathcal{K}_{r+2}$. Let $\mathcal{B}=\mathcal{A} \cap \mathcal{K}_{r+2}$. Suppose that rank $X>r$ for all $X \in \mathcal{B}$.

For every $X \in \partial \mathcal{B}$ we also have $X \in \partial \mathcal{K}_{r+2}$ and so rank $X<r+2$ (see Section 3.1). Since we assumed that $\operatorname{rank} X>r$ for all $X \in \mathcal{B}$, we must have $\operatorname{rank} X=r+1$ for each $X \in \partial \mathcal{B}$, so $\operatorname{dim}(\operatorname{Nul} X)=1$. The correspondence $X \longmapsto \operatorname{Nul} X$ defines a map $\psi: \partial \mathcal{B} \longrightarrow \mathbb{P}\left(\mathbb{R}^{r+2}\right)$, where $\mathbb{P}\left(\mathbb{R}^{r+2}\right)=\mathbb{R} P^{r+1}$ is the projective space of all lines through the origin in $\mathbb{R}^{r+2}$. Clearly, the map $\psi$ is continuous.

Now we claim that there exist two distinct points $X_{1}, X_{2} \in \partial \mathcal{B}$ such that Nul $X_{1}=$ Nul $X_{2}=\ell$ for some one-dimensional subspace $\ell \subset \mathbb{R}^{r+2}$. Suppose that this is not the case. Then $\psi: \partial B \longrightarrow \mathbb{R} P^{r+1}$ is an embedding. We note that since $\mathcal{A} \cap\left(\right.$ int $\left.\mathcal{K}_{r+2}\right) \neq \emptyset$, the set $\mathcal{B}$ is an $(r+2)$-dimensional convex body and the boundary $\partial \mathcal{B}$ is homeomorphic to the sphere $\mathbb{S}^{r+1}$. However, $\mathbb{S}^{r+1}$ cannot be embedded into $\mathbb{R} P^{r+1}$ for $r>0$. Indeed, since $\mathbb{S}^{r+1}$ and $\mathbb{R} \mathbb{P}^{r+1}$ are equidimensional manifolds, $\mathbb{S}^{r+1}$ is compact and $\mathbb{R} P^{r+1}$ is connected, the Invariance of Domain Theorem (see, for example, Chapter III, Section 6 of Massey, 1980) implies that such an embedding would have been a homeomorphism between $\mathbb{S}^{r+1}$ and $\mathbb{R} P^{r+1}$, which is a contradiction, since $\mathbb{S}^{r+1}$ and $\mathbb{R} \mathbb{P}^{r+1}$ are not homeomorphic for $r \geq 1$.

So, we proved that there exist two distinct points $X_{1}, X_{2} \in \partial \mathcal{B}$ such that $\mathrm{Nul} X_{1}=$ Nul $X_{2}=\ell$ for some one-dimensional subspace $\ell \subset \mathbb{R}^{r+2}$. Therefore, $X_{1}, X_{2} \in$ $\mathcal{F}_{\ell}$ for the face $\mathcal{F}_{\ell}$ of $\mathcal{K}_{r+2}$ (see Section 3.1). The intersection of the straight line $\left\{\tau X_{1}+(1-\tau) X_{2}: \tau \in \mathbb{R}\right\}$ passing through $X_{1}$ and $X_{2}$ with the face $\mathcal{F}_{\ell}$ is an 
interval $\left[Y_{1}, Y_{2}\right]$ with $Y_{1}, Y_{2} \in \partial \mathcal{F}_{\ell}$. Hence rank $Y_{1}, Y_{2} \leq r$ (see Section 3.1) and $Y_{1}, Y_{2} \in \mathcal{A} \cap \mathcal{K}_{r+2}$. The obtained contradiction shows that rank $X \leq r$ for some $X \in$ $\mathcal{K}_{r+2} \cap \mathcal{A}$.

Proof of Theorem 1.2. As in Section 31.5 of Deza and Laurent (1997), the intersection $\mathcal{K}_{n} \cap \mathcal{A}$ has an extreme point $Y$ and the rank of every such point is at most $r+1$, so codim Nul $Y \leq r+1$. We choose a subspace $\mathcal{L}$ such that $\mathcal{L} \subset \operatorname{Nul} Y$ and $\operatorname{codim} \mathcal{L}=r+2$. Let $\mathcal{F}_{\mathcal{L}}$ be the corresponding face of $\mathcal{K}_{n}$. Hence $Y \in \mathcal{F}_{\mathcal{L}}$ and $\mathcal{F}_{\mathcal{L}} \cap \mathcal{A} \neq \emptyset$. Since there is a rank-preserving isometry between $\mathcal{K}_{r+2}$ and $\mathcal{F}_{\mathcal{L}}$, by Lemma 3.2, it follows that there is an $X \in \mathcal{F}_{\mathcal{L}} \cap \mathcal{A}$ such that $\operatorname{rank} X \leq r$.

Remark. Our proof exploited in a simple minded way the fact that, as described in Section 3.1, the poset of faces of $\mathcal{K}_{n}$ is isomorphic to the poset of all subspaces of $\mathbb{R}^{n}$. It is our belief, however, that this fundamental fact will lead to deeper consequences in the future.

\section{Related Results from Matrix Theory}

Theorem 1.2 is equivalent to some results known in the literature. One can show that Theorem 1.2 is equivalent to the following result from Au-Yeung and Poon (1979) and Poon (1994).

(4.1) Theorem. Fix a number $r \geq 1$, a number $k \leq\left(\begin{array}{c}r+2 \\ 2\end{array}\right)-1$, and a number $n \geq$ $r+2$. Let $q_{1}, \ldots, q_{k}: \mathbb{R}^{n} \longrightarrow \mathbb{R}$ be quadratic forms and let $\varphi: \mathbb{R}^{n} \longrightarrow \mathbb{R}^{k}$ be the corresponding quadratic map, $\varphi(x)=\left(q_{1}(x), \ldots, q_{k}(x)\right)$.

Let $\mathbb{S}^{n-1}=\left\{x \in \mathbb{R}^{n}:\|x\|=1\right\}$ be the unit sphere. Then every point from conv $\varphi\left(\mathbb{S}^{n-1}\right)$ can be represented as a convex combination of $r$ (not necessarily distinct) points from $\varphi\left(\mathbb{S}^{n-1}\right)$.

In turn, it is shown in Au-Yeung and Poon (1979) that Theorem 4.1 is equivalent to the following (unpublished) result of Bohnenblust:

(4.2) Theorem. Suppose that $r>0$ and $n \geq r+2$. Let $\mathcal{U} \subset S_{\text {Sym }}$ be a subspace such that $\operatorname{dim} \mathcal{U}<\left(\begin{array}{c}r+2 \\ 2\end{array}\right)-1$. Suppose, further, that the following condition is satisfied: whenever for some vectors $x_{1}, \ldots, x_{r} \in \mathbb{R}^{n}$ one has

$$
\sum_{i=1}^{r} x_{i}^{t} A x_{i}=0 \quad \text { for all } \quad A \in \mathcal{U}
$$

one must have $x_{i}=0$ for $i=1, \ldots, r$. Then $\mathcal{U}$ contains a positive definite matrix.

Finally, Friedland and Loewy (1976) proved that Theorem 4.2 is equivalent to the following result. 
(4.3) Theorem. Suppose that $2 \leq r \leq n-1$. Let $\mathcal{W} \subset$ Sym $_{n}$ be a subspace such that $\operatorname{dim} \mathcal{W} \geq(r-1)(2 n-r+2) / 2$. Then $W$ contains a nonzero matrix whose largest eigenvalue is at least of multiplicity $r$.

To complete the circle, we sketch the proof of equivalence of Theorems 1.2 and 4.1. Theorem $1.2 \Longrightarrow$ Theorem 4.1 .

In the context of Theorem 4.1, let $y=\left(\eta_{1}, \ldots, \eta_{k}\right)$ be a point from the convex hull of $\varphi\left(\mathbb{S}^{n-1}\right)$. Hence $y=\alpha_{1} \varphi\left(x_{1}\right)+\cdots+\alpha_{m} \varphi\left(x_{m}\right)$ for some vectors $x_{1}, \ldots, x_{m} \in \mathbb{S}^{n-1}$ and some nonnegative $\alpha_{1}, \ldots, \alpha_{m}$ such that $\alpha_{1}+\cdots+\alpha_{m}=1$. Let $Q_{1}, \ldots, Q_{k}$ be the matrices of the forms $q_{1}, \ldots, q_{k}$ so that $q_{i}(x)=x^{t} Q_{i} x$. Let $X=\alpha_{1} x_{1} x_{1}^{t}+\cdots+\alpha_{m} x_{m} x_{m}^{t}$. Then $X$ is a positive semidefinite matrix satisfying $k$ affine constraints $\operatorname{tr}\left(Q_{i} X\right)=\eta_{i}$ for $i=1, \ldots, k$ and one additional constraint $\operatorname{tr}(X)=1$, which makes the set of all feasible positive semidefinite matrices bounded. Applying Theorem 1.2, we conclude that there exists a positive semidefinite matrix $X^{\prime}$ satisfying the same set of constraints and such that rank $X^{\prime} \leq r$. Such a matrix can be decomposed $X^{\prime}=\alpha_{1} x_{1}^{\prime} x_{1}^{\prime t}+\cdots+\alpha_{r} x_{r}^{\prime} x_{r}^{\prime t}$, which gives rise to a representation of $y$ as a convex combination of $\varphi\left(x_{i}^{\prime}\right), i=1, \ldots, r$.

Theorem $4.1 \Longrightarrow$ Theorem 1.2.

Reversing the above reasoning, one can show that Theorem 1.2 holds provided the subspace $\mathcal{A}$ lies in the affine hyperplane $\operatorname{tr}(X)=1$. A straightforward, but lengthy, argument deduces the general case of Theorem 1.2 from there.

\section{References}

1. A. Alfakih and H. Wolkowicz, On the embeddability of weighted graphs in Euclidean spaces, preprint, CORR 98-12, Department of Combinatorics and Optimization, University of Waterloo, Waterloo, Ontario 1998.

2. F. Alizadeh, J.-P.A. Haeberly, and M.L. Overton, Complementarity and nondegeneracy in semidefinite programming, Mathematical Programming, 77(2) (1997), 111-128.

3. E. Anderson and P. Nash, Linear Programming in Infinite-Dimensional Spaces. Theory and Applications, Wiley-Interscience, Chichester, 1987.

4. Y.H. Au-Yeung and Y.T. Poon, A remark on the convexity and positive definiteness concerning Hermitian matrices, Southeast Asian Bulletin of Mathematics, 3(2) (1979), 85-92.

5. A. Barvinok, Problems of distance geometry and convex properties of quadratic maps, Discrete \& Computational Geometry, 13 (1995), 189-202.

6. M. Deza and M. Laurent, Geometry of Cuts and Metrics, Algorithms and Combinatorics, Vol. 15, SpringerVerlag, Berlin, 1997.

7. S. Friedland and R. Loewy, Subspaces of symmetric matrices containing matrices with a multiple first eigenvalue, Pacific Journal of Mathematics, 62 (1976), 389-399.

8. M. Laurent, A tour d'horizon on positive semidefinite and Euclidean distance matrix completion problems, in: Topics in Semidefinite and Interior-Point Methods (Toronto, Ontario, 1996), pp. 51-76, Fields Institute Communication, Vol. 18, American Mathematical Society, Providence, RI, 1998.

9. W.S. Massey, Singular Homology Theory, Graduate Texts in Mathematics, Vol. 70, Springer-Verlag, New York, 1980.

10. G. Pataki, Cone-LP's and semidefinite programs: geometry and a simplex-type method, in: Integer Programming and Combinatorial Optimization (Vancouver, BC, 1996), pp. 162-174, Lecture Notes in Computer Science, Vol. 1084, Springer-Verlag, Berlin, 1996.

11. G. Pataki, On the rank of extreme matrices in semidefinite programs and the multiplicity of optimal eigenvalues, Mathematics of Operations Research, 23 (1998), 339-358. 
12. Y.T. Poon, On the convex hull of the multiform numerical range, Linear and Multilinear Algebra, 37(1-3) (1994), 221-223.

13. L. Vandenberghe and S. Boyd, Semidefinite programming, SIAM Review 38(1) (1996), 49-95.

14. R. Webster, Convexity, Oxford Science Publications, The Clarendon Press, Oxford University Press, New York, 1994.

Received July 8, 1999, and in revised form January 20, 2000, and May 9, 2000.

Online publication September 22, 2000. 